\title{
The Application of MATLAB Serial Interface Communication Technology in Cylinder Displacement Acquisition System
}

\author{
Wan $\mathrm{Xu}^{1, \mathrm{a}^{*}}$, Feiyue Chen ${ }^{1, \mathrm{~b}}$ and Jingang Liu ${ }^{1,2, \mathrm{c}}$ \\ ${ }^{1}$ College of Information Engineering, Capital Normal University, Beijing, China; \\ ${ }^{2}$ Institute of Computing Technology Chinese Academy of Sciences, Beijing, China; \\ axw_yatfg@163.com, booodcfy@126.com, cliujg2000@163.com
}

Keywords: MATLAB simulation; Serial communication; Data acquisition; Filter design

\begin{abstract}
In order to achieve the purpose of real-time monitoring of cylinder displacement, this paper takes the PC machine as the core and the RS232 serial communication interface as the research object. According to the electrical characteristics of the RS232 standard, combined with its own characteristics, the principle of ultrasonic distance measurement is used to design the hardware circuit of data acquisition, level conversion and data transmission. With MATLAB as the control system development platform, real-time data processing, graphical display processing results. The system interface is simple, easy to operate, quickly and accurately to draw the displacement curve. It not only improves the speed of data processing, but also provides a good platform for the development of two times and effectively saving the development cost. This hardware realization method is simple and has certain practical value.
\end{abstract}

\section{Introduction}

With the development and applications of computer systems, a number of complex systems have sprung up in all kinds of instrumentation, industrial control and automation field. And with the addition of system function, the amount of data being processed by computer is gradually increased.The communication between each system module and computers is also becoming more and more important. This paper is aimed at the pneumatic system for the data acquisition and processing. Pneumatic driving system has been widely adopted in the field of modern control because of its simple structure, low cost, non-pollution, and prompt action, etc. Currently there are a lot of the implementations of the cylinder displacement acquisition system, such as Visual $\mathrm{C}++$, $V B, . N E T$ and so on. Although these methods are simple, but they have the poor data processing ability and are lack of flexibility,these determine that current methods can't satisfy certain requirements and leads to high costs for the secondary development.

MATLAB has powerful data processing ability, which is widely used in signal processing, automatic control, the algorithm simulation and other fields, and can simultaneously combine its built-in serial communication module for real-time processing to the acquired data, then make the data acquisition system to get fast and accurate processing [1]. Its application in the field of algorithm simulation and static data processing is relatively mature, but in the practical pneumatic control areas its application is rare. In general, acquisition system makes mutual information to computer using serial communication, and MATLAB can read and manipulate the serial data through the establishment of serial objects [2]. In summary, this paper designed a cylinder positioning real-time acquisition system based on MATLAB GUI serial communication. The system not only expanded the range of MATLAB in practical application, but also found a reliable, efficient and stable way for real-time processing of data.

\section{General Design of the System}

The system includes upper and lower computer parts, of which the upper computer using MATLAB to operate a PC serial port for the data reading, processing and graphical displaying; Using the powerful computing ability of MATLAB, the cylinder's position signal can be calculated with some 
control algorithm, get the proper controlled quantity, and then send operation instructions to lower machine. This is beneficial to use simulation algorithm to control the basement effectively and directly and greatly shorten the time of the second development.

Cylinder position signal is acquired by lower machine through displacement sensor of SCM (single chip microcomputer) and transferred to PC through serial port, then the cylinder position can be adjusted by performing instructions received from the PC.

\section{Summary of Serial Communication and Implementation}

Data communication is the most important component of this system. So using a reliable and practical method of communication is the key part of the whole system. The communication of SCM with PC uses standard RS232 serial port communication protocol, because to be compared with parallel communication serial communication has followed merits, such as less wiring between devices, small cost, strong anti-interference ability, long transmission distance and so on. In accordance with the direction of data flow serial communication can be divided into three transmission modes: simplex, half-duplex and full-duplex[3]. Besides of the above-mentioned mode, there are other classifications, for example, it can be divided into asynchronous communication and synchronous communication. According to the system requirements, the selection is an asynchronous full-duplex mode that both sides of thecommunication can be sent and received at the same time with the sender and receiver in each side.

Protocol Analysis.It must abide by the same mode of data transmission to conduct the communication between two devices. This transport mode is the so-called communication protocol. The protocol defines the format and data bits of the data, etc. The following analysis against the format of data frame which is ruled by asynchronous serial communication protocol, and the frame format is shown in Fig. 1.

\begin{tabular}{c|c|c|c|c|c|c|c|c|c|c|c|c|c|c|c}
\hline$\ldots$ & 0 & $0 / 1$ & $0 / 1$ & $0 / 1$ & $0 / 1$ & $0 / 1$ & $0 / 1$ & $0 / 1$ & $0 / 1$ & $0 / 1$ & 1 & 1 & 1 & 0 & $\ldots$ \\
\hline & $\begin{array}{c}\text { start } \\
\text { bit }\end{array}$ & data bits & & $\begin{array}{c}\text { parity } \\
\text { check } \\
\text { bit }\end{array}$ & $\begin{array}{c}\text { stop } \\
\text { bit }\end{array}$ & $\begin{array}{c}\text { Free } \\
\text { bit }\end{array}$ & $\begin{array}{c}\text { start } \\
\text { bit }\end{array}$ &
\end{tabular}

Figure 1. The frame format of asynchronous serial communication

The Fig. 1 indicates that data frame of asynchronous serial communication is consisted of four components: start bit, data bits, parity check bit and stop bit[4]. Firstly the sending device sends a low level signal to play the role of synchronizing equipment in the data transfers, and then the receiver is ready to receive data after taking over the synchronization signal. Data bit are in the back of the start bit and its number can be 5,6,7,8. Parity check has three choices: even-parity check, odd-parity check and none, and it can only detect an odd number of data bits of error transmission but not correct the false data, so it is only to request a second sending if an error occurs. Stop bit is a character which marks the end of a data transfer, it could be 1, 1.5 or 2 high signals.

the Realization of Serial Communication Based on MATLAB. Serial communication interface of MATLAB provides a direct communication for the computer and external devices. The serial port device object is created by calling serial class of ICT(Instrument Control Toolbox), then users can set the object's properties, for example,the user can configure the serial port communication, use the control needle of serial port, read and write data, use events and callback, and record information to the document[5]. According to the serial port communication protocol, it requires the users to set the appropriate mode and parameters of the communication when using the serial port communication, so the following functions and procedures need to be established to achieve serial communication based on MATLAB:

(1) The creation of the serial port devices and objects

s_serialport=serial (com_num); 
Create a serial port object whose name is designated by com_numand then return the handle of this serial port object. If the object does not exist or is using, this object cannot connect to the serial device.

(2) The settings of the properties of serial port object

set(s_serialport, 'BaudRate',baud_num,'DataBits',data_len,'Stop

-Bits', stop_len,'Parity',parity_num);

Here set the baud rate, data bits, stop bits and parity bits which are specified by serial port communication protocol. But this is just to meet the most basic communication properties of the agreement, in accordance with the provisions of MATLAB, there is also need to set a callback function for receiving, the threshold of byte received from serial port and the mode of data reading.

$$
\begin{aligned}
& \text { s_serialport.BytesAvailableFcnMode='byte'; } \\
& \text { s_serialport.BytesAvailableFcnCount }=4 ; \\
& \text { s_serialport.BytesAvailableFcn= }\{\text { @serialport_break,handles }\} \text {; }
\end{aligned}
$$

Based on the requirements of system, here sets the mode of reading data for byte, the threshold of byte received from serial port for 4 and the callback function of data processing for serialport_break.

(3) The opening of the serial communication port

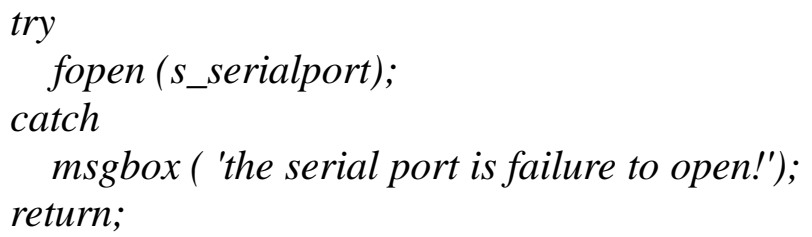

If the serial port is failure to open, here will display a pop-up tip to make the developers can debug easily.

(4) The receiving and sending of serial port data

When above configurations of serial port are successful,the data can be sent and received. It commonly uses such as fgetl, fgets, fread, fscanffunctions to read data from serial devices[6], and the difference between these functions is that the type of reading data is different. In order to facilitate post-processing of data, here chooses fread function whose data type is binary system data to read data from the serial devices. Use $r e c=f r e a d\left(s \_s e r i a l p o r t, 4\right)$ for data reading.

There can use fprintfand fwritefunction to write data to the device, the two functions' difference is that the types of written data are not same, fprintf uses ASCII code and fwrite uses binary. Similar to the way of reading, here also uses a binary manner to write, that is to say, fwrite function is used to do the data writing. Specific method is as follows: fwrite (s_serialport,sen).

(5) The disconnection and removing of serial port object

At the end of communication, when one serial port object is not necessary, $f c l o s e\left(s \_s e r i a l p o r t\right)$ should be used for breaking the connection between it and devices, delete(s_serialport) for removing it from memory, clear s_serialport for releasing its workspace in MATLAB.

The Realization of Serial Communication Based on SCM. In this paper, 51 series SCM are used as an example. 51 series has a programmable full-duplex serial communication interface, it not only can be used as UART (universal asynchronous receiver/transmitter), but also be used as synchronous shift registers. To control the serial communication interface, the following registers are needed to use.

SCON (serial control register), to set the mode of transmission and reception of serial communication, of which SMO, SMI can set serial port of SCM how to work; TI, RI respectively show as the interrupting flag of sending and receiving, and then one is set by hardware, zero is set 
by software after interruption.TMOD (timer/counter mode control register) is to be used for setting the working manner of timer $T O$ and $T 1$, and mode two (automatic loading eight counters) of which is generally employed in serial communication that it is using the timer's overflow to generate the baud rate.TCON (timer control register), of which TR1, TRO is separately used as the switch of timer $T 1$ and $T 0 ; T F 1$, TFO is used as the overflow flag, it definitely refers to set the corresponding position to one when there is the overflowing in timer $T 1$ and $T 0$, then turn to CPU for breaking off, and finally automatically clear these bits when the interruption is responded.

TheSetting of Baud Rate. Hardware circuit is a basic of serial communication, to achieve the communication between PC and SCM, here needs to set the rate of sender and receiver. The transmitting signal in communication line must be maintained to one consistent signal duration, which is decided by baud rate.Baud rate means data transfer rate, this represents the number of transmitting binary digits on the transmission line per second. Serial port of 51 series single-chip has four working modes, which are as follows: The baud rate of mode 0 and mode 2 is fixed; the baud rate of mode 1 and 3 is decided by the timer Tl's overflow. Here uses the timer $T 1$ which works under the automatic loading initial value model as baud rate generator. Using the different initial value to produce the different baud rate,and according to the internal structure of the single-chip the baud rate is calculated as follows:

Baud rate $\cong \frac{2^{S M O D}}{32} \times \frac{F O S C}{12 \times\left(2^{8}-X\right)}(1)$

The calculation formula of initial value $X$ can be obtained when the timer adopts mode 2 :

$$
\mathrm{X} \cong 2^{8}-\frac{F O S C \times(S M O D+1)}{384 \times \text { Baud rate }}(2)
$$

This design's baud rate is $9600 \mathrm{bps}$, SMOD is 0, FOSC is $11.059 \mathrm{MHz}$, then 0xFD can be calculated out as the initial value of TH1 and TL1 by Eq. 2.

\section{TheProcessing of Real-Time Signal}

With the above implementation method of serial communication, using MATLAB powerful data processing capabilities, the data received from SCM can be processed in real time, and the processing mainly includes filtering, noise processing and real-time display [7]. Since the cylinder movement belongs to the mechanical movement area,thus the filtering requirements for collected location information are relatively simple, here only discusses several classic algorithms for digital filtration.

TheMedian Filter Method.Taking $N$ sampling values in a row and arranging them in order of size, then taking the median as the valid value, doing above that can effectively filter out the interference of impulses generated by chance. For the determining of value $N$, it depends on the source of noise and the frequency, when the noise frequency is relatively high, selecting a smaller value of $N$ can achieve better results. If the noise frequency is low, that results in a small difference in the sample data, and then such a small value of $N$ is more difficult to filter spikes, so it is better to increase the value of $N$. The main noise frequency of this experiment is high, thus here sets the value of $N$ to 5 .

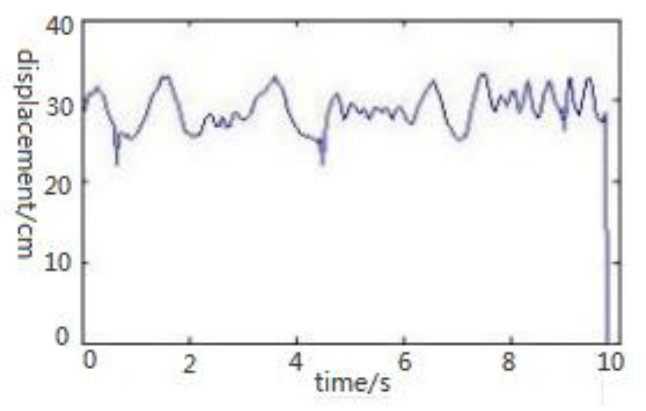

Figure 2. The original sampling data 


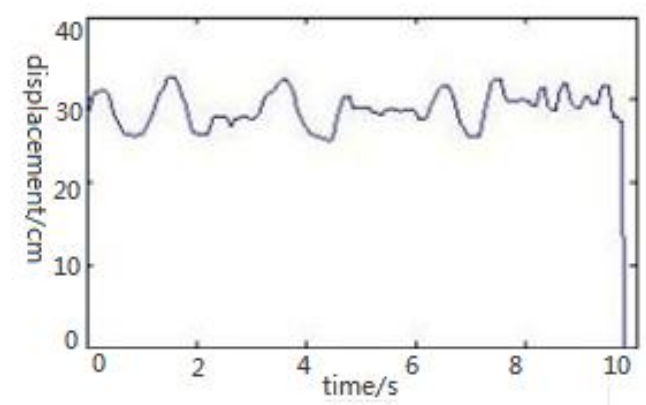

Figure 3. The result of median filter

Itcan be seen from Fig. 3 that median filtering has a certain effect on filtering of pulse noise in signals when compared with Fig. 2. But for some continuous fluctuation, the noise is not well filtered. Filtering effect is better in the signal period whose change of frequency is relatively high than in the signal period relatively low. So how to choose a suitable $\mathrm{N}$ becomes the key to achieve good results.

TheArithmetic Average Filter Method. Arithmetic average method is to perform arithmetic and average computations on $N$ continuous sampling data and get the result of the above calculation as the effective output of this sample [8]. And about value $N$,it not only determines the sensitivity and smoothness of the signal, but also determines the computational complexity. In order to compare the effect of this method with previous median filtering method, value $N$ is also taken 5 .

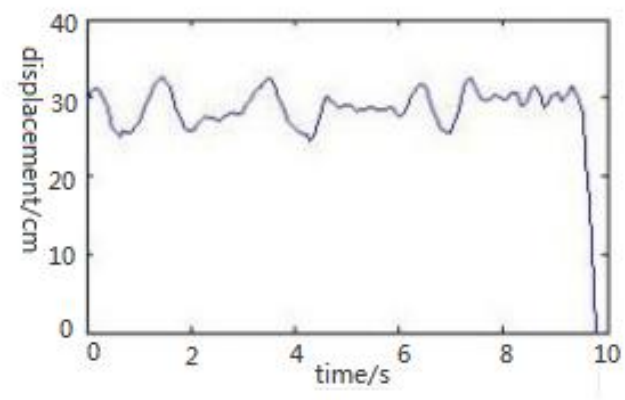

Figure 4. The result of arithmetic average filter

Fig. 4 clearly shows that the arithmetic mean filtering method effectively slashes the spikes, but doesn't have very good smoothing effect. Moreover, if value $N$ is increased, the computation will be also increased, but the sensitivity is reduced.

The Mean Median Filter Method.For $N$ continuous sampling data, firstly it is sorted by their value size, and then its maximum and minimum are took out.Finally the arithmetic mean of left data is calculated out as the effective value of this filter [9]. When value $N$ is three the method is median filter, so the range of $N$ should be greater than three. In theory, this filtering method has both advantages of the median filter and the mean filter, that is, this method should have good inhibitory effect on the impulse noise and modest random disturbance, but the actual effect may be less than the median filter adding the arithmetic average filter. 


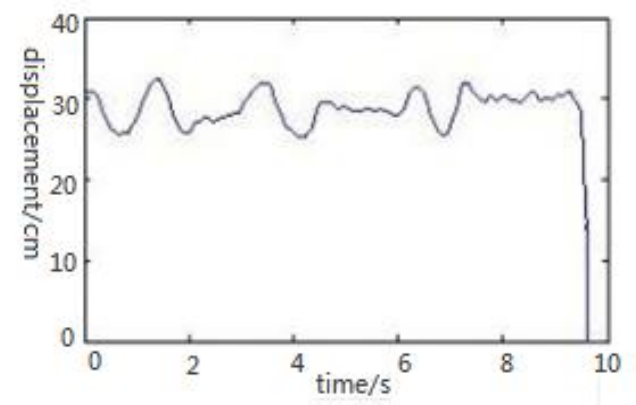

Figure 5. The result of mean median filter method

This time sets value $N$ to seven, it can be seen from Fig. 5 that the whole curve is relatively smooth, this method has good filtering effect on not only the high-frequency noise but also the relatively low frequency spikes.

The Limiting Amplitude Filter Method.First, the allowable maximum deviation between twice sampling is determined based on experience and is set to $A$. Then, when detect a new value every time: If the difference between this value and the last value is less than or equal to $A$, this value is effective; If the difference between this value and the last value is more than $A$, this value should be given up as an invalid value and be replaced by the last value. Determining the maximum deviation is very difficult when being used in practice. If selected $A$ is too small, the useful signal will be filtered;if selected $A$ is too large, the noise can't be filtered out. According to the actual situation, this experiment sets $A$ to three meters and the result is drawn in Fig. 6.

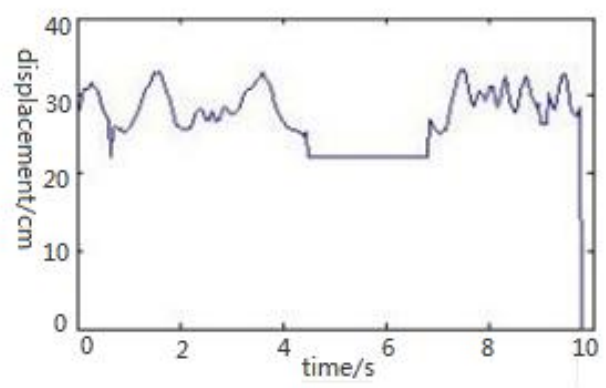

Figure 6. The result of limiting amplitude filter method

It is clear that limiting amplitude has some effect on filtering spikes from Fig.6, but because of the unreasonable setting of maximum deviation, obviously a part of useful information in the middle section of signal is lost compared with original signal,that is often called distortion.

The First-Order Lag Filter Method. The effect of digital filtering achieved by simulating hardware $R C$ filteris equivalent to a first-order $R C$ filter [10]. Following formulas can be evolved through the differential equation of first-order $R C$ filter.

$$
\begin{aligned}
& \frac{Y(z)}{X(z)}=\frac{T}{R C\left(1-z^{-1}\right)+T}(3) \\
& Y(n)=\frac{T}{T+R C} X(n)+\frac{R C}{T+R C} Y(n-1)(4)
\end{aligned}
$$

Eq. 3 is the transfer function of first-order $R C$ filter in $S$ domain, and turning into time domain the difference equation is Eq. 4, so the evolved formula of first-order lag filter is:

$$
\mathrm{Y}(n)=a * y(n-1)+(1-a) * x(n) \quad a=\frac{R C}{T+R C}(5)
$$

Among above equations, $T$ is the sampling period, $R C$ is time constant for the filter, $a$ is called filter coefficient. The bigger the $a$, the more steady the filtering result and the lower the sensitivity; the smaller the $a$, the less steady the filtering result and the higher the sensitivity. 


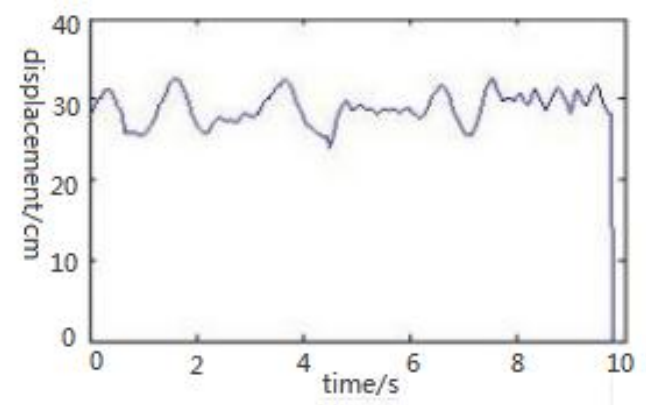

Figure 7. The result of first-order lag filter method

According to the characteristics of this experiment, $a$, the coefficient of filter, is set to 0.6. As can be seen from Fig. 7, the whole signal is smooth, but due to the reason for selecting filter coefficients, there are some spikes are not able to well filtered. Therefore, there is a median filter added after the first-order lag filter to eliminate some of the small amplitude filtering, and the result shown in Fig. 8.

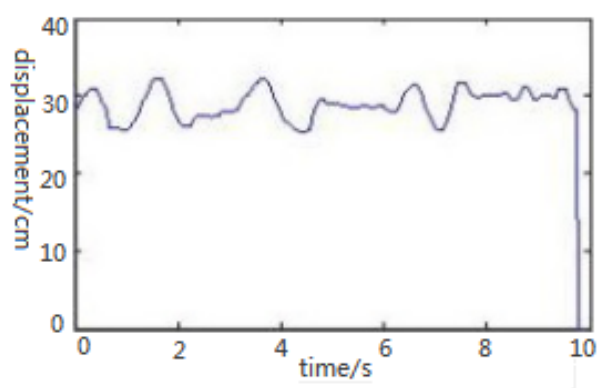

Figure 8. The result of first-order lag filter with median filter method

According to the results above, as well as many of the experiments, and ultimately the first-order lag filter with a median filter is selected as the filter of the present collection system.

\section{Conclusion}

In summary, MATLAB is characterized by its simplicity and convenience in collecting serial data. It not only has high efficiency and stability in data processing, but also benefits the secondary development by using its powerful functions of a digital filter. And compared with the data capture system that other software platforms have developed, it takes up fewer resources, shortens the development cycle, saves the development costs, and extends its application to other areas.

\section{References}

[1] Y.Chen:Design of ARM-based Portable Multi-gas Detector for Coal Mine (MS.Xi'an University of Science and Technology, China 2013), p.15-20.

[2] X. Liang: Science and Technology Information (Science and Research), (2008)No.9, p.44-45.

[3] Y.P. Zhao: Microcomputer Information, Vol.22 (2006)No.1, p.111-112.

[4] W.W. Huang, G.P. Shao: Microcomputer Information, Vol.21 (2005) No.4, p.132-133.

[5] H. Y: Design of Laser Doppler Velocimeter (MS.Chongqing University, China 2012), p.12-18.

[6] H.F. Luo:MATLAB GUI design learning Notes(Beihang University Publication, China 2011), p.30-36.

[7] W. Cheng, W. B. Cheng and C.P. Li: Electronic Measurement Technology, Vol.31 (2008) No.8, p.92-94.

[8] Y.L. Zhao: Journal of Lanzhou Institute of Technology, Vol.18 (2011) No.6, p.4-6.

[9] Q.H. Wang andQ.S. Wang: Measurement Technology, (2003)No.4, p.53-55.

[10]B. Wang: Mining Engineering, Vol.7 (2009) No.3, p.55-57. 